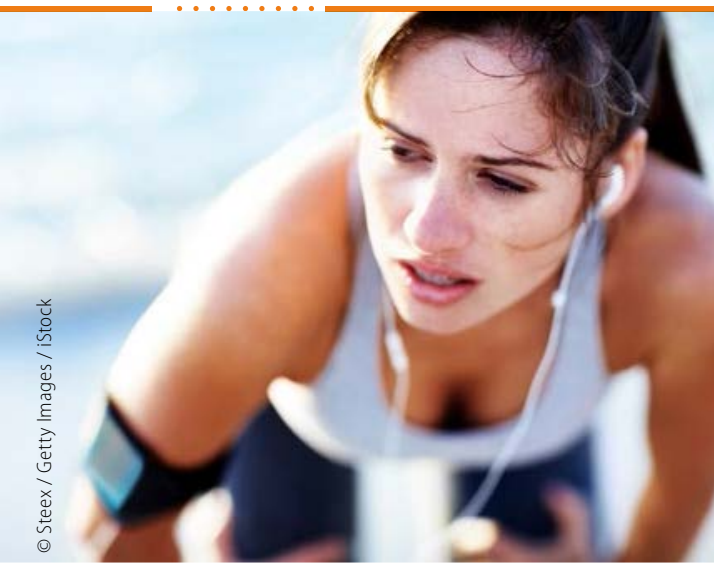

Leistungsminderung, Depressionen, Schlafstörung oder Appetitlosigkeit bei sportlichen Patienten können auf ein Übertrainingssyndrom hinweisen.

Ausdauersport zählt zu den wichtigsten präventiven Maßnahmen überhaupt. Wird das Training allerdings übertrieben, kann aus dem gesundheitlichen Nutzen auch ein Schaden werden. Leistungsminderung, Schlafstörungen, Appetitlosigkeit Nachtschweiß, Kopfschmerzen, Depressionen oder erhöhte Infektanfälligkeit - solche Beschwerden können auf ein Übertrainingssyndrom hinweisen, berichtete PD Dr. Thomas Bobbert, von der Charité in

Sportler körperlich und psychisch erschöpft

\title{
Denken Sie auch an das Übertrainingssyndrom!
}

\begin{abstract}
Hohe Trainingsintensitäten oder zu monotone Belastungen können bei Sportlern zum Phänomen des Übertrainings führen.
\end{abstract}

Berlin beim Internistenkongress. Es könne ausgelöst werden durch hohe Trainingsintensitäten über einen längeren Zeitraum, vor allem im hochintensiven Ausdauerbereich, zu monotone Belastungen, zu schnelle Trainingsaufnahme nach Infekten oder auch einseitige Ernährung.

\section{Auch Hobbysportler können betroffen sein}

Betroffen seien vor allem Leistungssportler, sagte Bobbert, aber auch Hobbysportler mit sehr hoher Trainingsintensität könnten ein Übertrainingssyndrom entwickeln und ab und $\mathrm{zu}$ in der Allgemeinarztpraxis auftauchen.
Wichtig sei es, bei Sportlern an ein solches Syndrom zu denken, betonte der Endokrinologe. Wegen der unspezifischen Symptome sei es schwer zu diagnostizieren. $\mathrm{Zu}$ hormonellen Auffälligkeiten könnten niedrige Testosteronwerte, oft in Verbindung mit Libidoverlust, sowie anomale Spiegel an Schilddrüsenhormonen und Wachstumshormon zählen. Bis sich Sportler von einem Übertrainingssyndrom erholen, kann es nach Angaben von Bobbert Wochen dauern.

Bobbert T: Sport in der Endokrinologie. 123. Kongress der Deutschen Gesellschaft für Innere Medizin (DGIM), 01. Mai 2017, Mannheim

\section{Keine Angst vor Sport bei multimorbiden Arthrose-Kranken}

\author{
Mit einem maßgeschneiderten Trainingsprogramm \\ lassen sich offenbar auch bei Patienten mit Begleit- \\ erkrankungen Arthrose-bedingte Beschwerden \\ sicher und effektiv lindern.
}

Bewegung ist ein wichtiger Bestandteil der Arthrosetherapie. Bei Patienten, die neben ihrer Kniearthrose noch ein Herzoder Lungenleiden haben oder zusätzlich an Diabetes erkrankt sind, wird jedoch aufgrund der eingeschränkten körperlichen Belastbarkeit und aus Angst vor trainingsbedingten Zwischenfällen oft darauf verzichtet.

Niederländische Rehabilitationsmediziner und Orthopäden haben 126 Probanden mit Gonarthrose in zwei Gruppen randomisiert. Alle litten zusätzlich an mindestens einer Begleiterkrankung, da- runter koronare Herzkrankheit, Diabetes, chronisch obstruktive Lungenerkrankung oder Adipositas. Die Patienten der Interventionsgruppe trainierten 20 Wochen lang unter Anleitung eines Physiotherapeuten Kraft und Ausdauer. Die Trainingsintensität wurde anhand der BorgSkala beurteilt und individuell an den jeweiligen Gesundheitszustand adaptiert. Die Patienten der Kontrollgruppe erhielten lediglich ihre gewohnte Medikation.

Die körperliche Leistungsfähigkeit wurde bei allen Probanden zu Studienbeginn sowie nach 10, 20 und schließlich nach 32 Wochen anhand der Subskala „physische Funktion“ des Western Ontario and McMaster Universities Osteoarthritis-Indexes (WOMAC-pf) und des 6-Minuten-Geh-Tests (6-MWT) beurteilt.

\section{Training war sicher und effektiv}

Die Probanden der Interventionsgruppe schnitten im Studienverlauf signifikant besser ab als die der Kontrollgruppe. Nach drei Monaten hatten sich die Teilnehmer der Interventionsgruppe auf der WOMAC-pf-Skala im Schnitt um 11,6 Punkte (33\%) verbessert, im 6-MWT um $59 \mathrm{Me}$ ter (15\%). Schwerwiegende trainingsbedingte Zwischenfälle traten nicht auf. $d k$

De Rooij M et al. Arthritis Care Res 2017; doi: 10.1002/acr.23013 